\title{
TAMAÑO, ESTRUCTURA Y DISTRIBUCIÓN ESTACIONAL DE POBLACIONES DE AVES ACUÁTICAS EN EL FIORDO ÚLTIMA ESPERANZA Y CANAL SEÑORET, REGIÓN DE MAGALLANES
}

\author{
SIZE, STRUCTURE AND SEASONAL DISTRIBUTION OF AQUATIC BIRDS POPULATIONS \\ FROM ÚLTIMA ESPERANZA SOUND AND SEÑORET CHANNEL, MAGELLAN REGION
}

Gladys Garay¹, Oscar Guineo${ }^{1}$ Erika Mutschke² \& Carlos Ríos ${ }^{2,3}$

\begin{abstract}
Between September 2001 and September 2002, Ultima Esperanza fjord and Señoret Channel were surveyed, sailing from Puerto Natales. Information on abundance, structure and distribution of aquatic and land birds observed during the sail is presented. We found 30 species of aquatic birds and 3 land birds.: 1 In Danger, 3 Vulnerables and 1 Insufficiently known. Our analysis shows that these fjords contained one of the biggest winter abundance of birds of the region and is a nesting area for many species. Furthermore, we proposed that the fjord constitute a core area for aquatic birds in summer when fresh water lagoons and ponds get dry. Finally, we make special reference to the need to implement a management plan in the area.
\end{abstract}

Key words: Sea birds, land birds, Southern fjords, aquatic birds, conservation, marine park, subantarctic, Magallanes

\section{RESUMEN}

El fiordo Última Esperanza y canal Señoret forman un ecosistema marino cuya característica biótica esencial es la presencia de una rica fauna vertebrada. Hasta el momento no se habían realizado catastros, estudios o registros sistemáticos de las especies que allí habitan. En el presente trabajo, se reportan los primeros antecedentes referidos al tamaño, estructura y distribución estacional de las principales poblaciones de aves presentes en estos sectores. Los datos fueron tomados entre septiembre de $2001 \mathrm{y}$ septiembre de 2002. Se registró la presencia de 30 especies de aves acuáticas y 3 terrestres (asociadas al área). De la avifauna registrada, 5 especies han sido consideradas con problemas de conservación a nivel nacional: 1 En Peligro (coscoroba), 3 Vulnerables (cóndor, cisne de cuello negro, flamenco chileno)

\footnotetext{
Fundación Garay-Guineo, Punta Arenas, Chile. guineo.garay@gmail.com

Laboratorio de Ecología y Ciencias Ambientales, Instituto de la Patagonia, Universidad de Magallanes, Punta Arenas, Chile

3 Dirección de Programas Antárticos, Universidad de Magallanes, Punta Arenas, Chile
} 
y 1 Inadecuadamente conocida (quetru volador). Los antecedentes reunidos indican que el área de estudio es uno de los sitios de mayor concentración de aves de la región, durante la temporada de muda e invernada (otoño austral) y, además, es el sitio de nidificación para numerosas especies. Por otra parte, en la época de primavera y verano australes se localizaron colonias reproductivas de cormorán imperial, cormorán de las rocas y bandurrias. Las especiales características de las aguas del sistema estudiado pueden contribuir a explicar las altas concentraciones de aves en otoño-invierno, si se considera que todas las especies inmigrantes utilizan cuerpos de agua dulce interiores, los que se secan a fines del verano o se congelan a fines de otoño. En base a estos resultados, se recomienda brindar una protección adecuada al sistema estudiado.

Palabras clave: Fiordos australes, aves acuáticas, conservación, parque marino, subantártica, Magallanes.

\section{INTRODUCCIÓN}

La presencia de vida silvestre en áreas poco intervenidas, ya sea por la protección de los lugares en que se encuentran (e.g. Reservas, Monumentos Naturales y Parques Nacionales) o por la inaccesibilidad de los hábitats que la constituyen (e.g. canales y fiordos marinos del archipiélago patagónico), ha determinado la existencia de un foco de alta atracción turística en la provincia de Última Esperanza, región de Magallanes y Antártica Chilena. Esto está corroborado por datos del Servicio Nacional de Turismo que establecen que, dentro de la región, la provincia de Última Esperanza es el área más visitada por turistas extranjeros y nacionales.

Esta presión sobre los recursos escénicos y de vida silvestre ha aumentado sostenidamente en los últimos años y se refleja en el marcado interés por visitar el área del fiordo, incrementándose la organización de circuitos turísticos que lo conectan con el Parque Nacional Torres del Paine, a través del río Serrano (Garay \& Guineo 2003¹).

No obstante la reconocida presencia de numerosas especies de aves y mamíferos en la región (e.g. Venegas \& Jory 1979, Sielfeld \& Venegas 1980, Gibbons et al. 1998, Venegas et al. 2002), hasta ahora no se ha realizado un estudio sistemático que determine qué especies están presentes, cuál es su estatus poblacional y sus ciclos de abundancia a lo largo del tiempo y del espacio en áreas específicas como el seno de Última Esperanza. Esta información es especialmente relevante e importante no sólo por

\footnotetext{
Garay, G. \& O. Guineo 2003. Tamaño, estructura y distribución estacional de la población de aves y mamíferos presentes en el fiordo Última Esperanza y canal Señoret y características y calidad de sus aguas. Informe Inédito. 25 pp.
}

su implicancia para el desarrollo del turismo, sino que, además, por las actividades de acuicultura, especialmente de especies salmonídeas, que se está reactivando fuertemente en la región de Magallanes en los últimos años. Estas actividades productivas, unidas a las actividades más pasivas asociables al turismo, suelen generar impactos negativos sobre las características de los ecosistemas naturales si no se realizan bajo estrictas medidas de manejo. Las modificaciones que se generan en el ambiente pueden provocar, a mediano o corto plazo, por ejemplo la migración y/o mortalidad de la vida silvestre que allí existe y en otros tipos de servicios que pueden ofrecer los ecosistemas acuáticos, tal como se ha ejemplificado dramáticamente para otras regiones del país (e.g. Jara 2005, Soto \& Norambuena 2005).

En el presente trabajo se entregan los primeros antecedentes cuantitativos acerca de las poblaciones de aves acuáticas y terrestres de normal ocurrencia en el área de Última Esperanza. Los objetivos del trabajo fueron determinar el tamaño, estructura y distribución de la población de aves, presentes en el fiordo Última Esperanza y canal Señoret y establecer si estas características poblacionales cambian en las diversas estaciones del año. El estudio implicó la realización de un catastro de las especies de aves y mamíferos que habitan en el fiordo Última Esperanza y canal Señoret, durante un período de 1 año, con el fin de determinar los posibles cambios en la composición específica de esta fauna, elegir las especies principales, según criterio de presencia de colonias y estado de conservación de su población, y localizar las áreas de uso y el tiempo de utilización de ellas, por parte de las especies seleccionadas. 


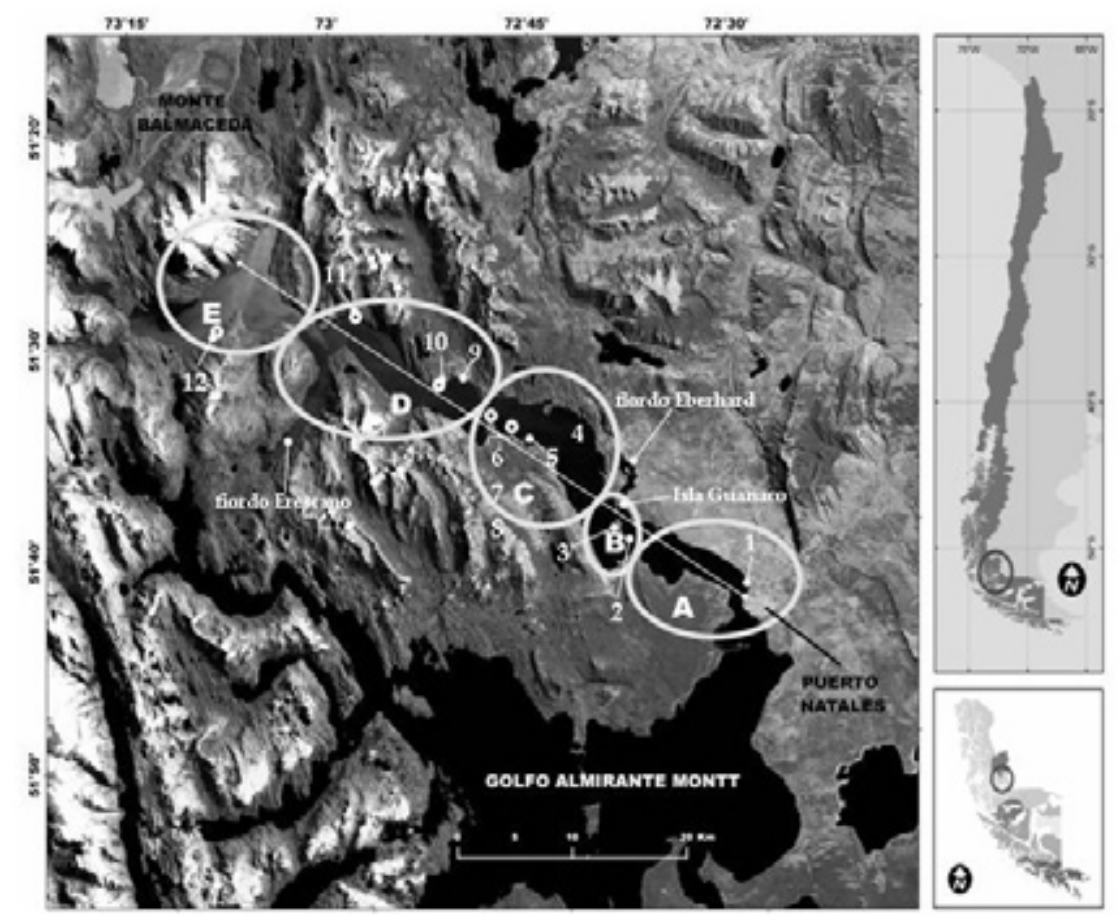

Fig. 1. Área de estudio que incluye el fiordo Última Esperanza y el canal Señoret. El trazo entre Puerto Natales y monte Balmaceda representa una distancia aproximada de $55 \mathrm{~km}$. Los números (112) indican las posiciones de muestreo y que se indican en la tabla 1 . Se incluyen algunos lugares de referencia y la sectorización (A-E; Véase tabla 1 para toponimia) para los censos generales.

\section{MATERIALES Y MÉTODOS}

\section{a) Área de Estudio}

El área de estudio incluyó el fiordo Última Esperanza y el canal Señoret (Fig. 1). Todas las áreas y distancias a que se hacen referencia, fueron calculadas a partir de una imagen satelital y con datos tomados in situ con un GPS Garmin II Plus (Datum WGS 84). Los sitios específicos y sus coordenadas geográficas se indican en la tabla 1.

El fiordo Última Esperanza tiene $190 \mathrm{~km}^{2} \mathrm{de}$ superficie (44 km de largo, $1.000 \mathrm{~m}$ a $4.200 \mathrm{~m}$ de ancho y profundidades entre 10 - $545 \mathrm{~m}$ ). Se extiende de SE a NW desde la isla Guanaco hasta el monte Balmaceda, limitando aquí con el Parque Nacional Bernardo O'Higgins. En este fiordo convergen otros dos fiordos, el Erezcano frente a la cascada Cóndores y el Eberhard frente a la isla Guanaco en su límite sur (de 10 y $9 \mathrm{~km}$ de largo respectivamente). Por el extremo noroeste desembocan el lago Azul y el lago Blanco y por el noreste el río Serrano, proveniente de la red hídrica del Parque Nacional Torres del Paine.
La primera mitad del fiordo se ubica entre terrenos ondulados y cerros que se elevan desde los 650 m (cerro Ballena) a 1.180 m (cerro Prat), cubiertos de matorrales (e.g. Chilliotrichum diffusum) y bosques (e.g. Nothofagus spp.). En el borde costero se presentan algunas ensenadas amplias y protegidas. El terreno se eleva hacia el NW en la segunda mitad del fiordo y sus aguas corren entre cerros de $1.500 \mathrm{~m}$ (cordillera Moore) y $2.035 \mathrm{~m}$ de altura (monte Balmaceda), con abruptos acantilados en al área más cercana al Balmaceda. La vegetación también va cambiando de matorrales a bosques, densos en algunos lugares y abiertos en otros, donde ha intervenido el hombre a través de la tala e incendios forestales. Una descripción detallada de la vegetación del área se encuentra en Pisano (1977).

El canal Señoret tiene aproximadamente 44 $\mathrm{km}^{2}$ de superficie y entre 2 - $30 \mathrm{~m}$ de profundidad. Limita al sur con el golfo Almirante Montt y al norte con la isla Guanaco, con una extensión de aproximadamente $11 \mathrm{~km}$ de largo. Se encuentra entre terrenos ondulados y de media altura, de 
TABLA 1. Localización geográfica de los sitios censados (1-12) para la cuantificación de la fauna presente en el fiordo Última Esperanza, situados de Sur a Norte en la figura 1. Datum WGS 84. Se incluyen los sectores (A-E) con las divisiones arbitrarias del área de estudio.

\begin{tabular}{llcc}
\hline \multicolumn{1}{c}{ Lugar } & \multicolumn{1}{c}{ Tipo de sitio } & Longitud O & Latitud S \\
\hline 1. Muelle Braun y Blanchard & colonia de Cormorán Imperial & $72^{\circ} 30.679^{\prime}$ & $51^{\circ} 43.432^{\prime}$ \\
2. Isla Rata & colonia mixta & $72^{\circ} 38.553^{\prime}$ & $51^{\circ} 40.580^{\prime}$ \\
3. Isla Lagartija & posadero de aves & $72^{\circ} 38.987^{\prime}$ & $51^{\circ} 40.094^{\prime}$ \\
4. Bahía Delfines & presencia de delfines y lobos & $72^{\circ} 44.066^{\prime}$ & $51^{\circ} 35.183^{\prime}$ \\
5. Islote Díaz & nidificación de bandurrias & $72^{\circ} 46.662^{\prime}$ & $51^{\circ} 35.352^{\prime}$ \\
6. S.E. Punta Barrosa & colonia Cormorán de las Rocas & $72^{\circ} 47.678^{\prime}$ & $51^{\circ} 35.021^{\prime}$ \\
7. Punta Barrosa & colonia Cormorán Imperial (2001) & $72^{\circ} 49.463^{\prime}$ & $51^{\circ} 34.331^{\prime}$ \\
8. Punta Barrosa & colonia Cormorán Imperial (2002) & $72^{\circ} 49.106^{\prime}$ & $51^{\circ} 34.559^{\prime}$ \\
9. Estancia Perales & área pelecha de caiquén y canquén & $72^{\circ} 51.203^{\prime}$ & $51^{\circ} 32.503^{\prime}$ \\
10. Barranca los Lobos & apostadero de lobo de un pelo & $72^{\circ} 53.964^{\prime}$ & $51^{\circ} 32.624^{\prime}$ \\
11. Acantilado de Cóndores & posadero de cóndores & $73^{\circ} 00.359^{\prime}$ & $51^{\circ} 28.974^{\prime}$ \\
12. Lobería Balmaceda & paridero de lobos de un pelo & $73^{\circ} 09.704^{\prime}$ & $51^{\circ} 29.358^{\prime}$
\end{tabular}
A. Canal Señoret
B. Área de unión del canal
Señoret y boca del fiordo
C. Bahía Delfines
D. Acantilados (Barranca de Lobos y de los Cóndores)
E. Cabecera del fiordo (unión del río Serrano y fiordo)

vegetación estepárica y matorrales, con costas y ensenadas semiprotegidas y poco profundas. Una descripción detallada de la vegetación del área se encuentra en Pisano (1977). Parte de la costa este se encuentra frente a la ciudad de Puerto Natales, al ex frigorífico Bories y al Puerto Prat, mientras que la costa oeste está influenciada por los campos ganaderos de la península Antonio Varas. En ambas costas hay sectores autorizados para el cultivo de salmones.

\section{b) Método de trabajo en terreno}

Se realizaron censos por conteo directo, utilizando binoculares de largo alcance (Tasco 10-30x50 mm.) y un contador de unidades, recorriendo un total de $53 \mathrm{~km}$ desde Puerto Natales hasta la base del monte Balmaceda en el yate/motor "Alberto de Agostini". Esta es una embarcación de $21 \mathrm{~m}$ de eslora, 5,5 m de manga, 2 motores de $360 \mathrm{HP} \mathrm{y}$ capacidad para 70 pasajeros. La velocidad normal de recorrido fue de aproximadamente $17 \mathrm{~km} / \mathrm{h}$ (9 millas náuticas). La altura de observación en la cubierta superior fue de $6 \mathrm{~m}$.
Los censos se realizaron cada 15 días, entre el 19 de septiembre de 2001 y el 29 de septiembre de 2002. El conteo se realizó durante el viaje de ida (entre las 8:00 y 12:00 h) y la distancia de observación varió entre 5 m y 3.500 m. Desde fines de mayo hasta fines de julio (estaciones de otoño e invierno australes), los censos se hicieron durante el viaje de regreso, entre las 13:00 y 16:00 $h$, debido a la insuficiencia de luz hasta pasada las 10:00 AM. En cada censo participaron dos especialistas, uno ubicado a babor y otro a estribor de la embarcación.

En las áreas de las colonias de reproducción de los cormoranes, se tomaron fotografías en cada control para realizar el conteo posterior bajo una lupa estereoscópica. También se llevó registro fotográfico de las otras áreas de interés, como lugares de alta concentración de aves en el mar en los meses de invierno.

Sobre la base del primer registro, se eligieron las especies que se mantendrían bajo seguimiento en los dos censos mensuales, durante un año. Se descartaron aquellas especies de coloración oscura y tamaño pequeño, que se mimetizan o pierden 
en el ambiente a la distancia a la cual se observan desde la lancha.

Para determinar las áreas de uso de estas especies, se hizo una zonificación del fiordo subdividiéndolo en 5 sectores (Fig. 1). Estos sectores fueron seleccionados básicamente por características geográficas que pudieran estar generando unidades diferenciadas de hábitat, tales como angosturas, (1.000 $\mathrm{m}$ la menor), ensenadas (4.200 $\mathrm{m}$ la mayor), islotes, acantilados, entre otros.

Adicionalmente, se realizaron 3 censos generales correspondientes a las estaciones de verano, otoño e invierno. El censo de verano se realizó entre el 27 y 28 de enero, recorriendo el fiordo Última Esperanza en la lancha habitual. Luego por tierra y en vehículo, se recorrió el borde costero este del canal Señoret a lo largo de $10 \mathrm{~km}$ por el norte y sur (desde Puerto Bories hasta punta Galón, frente al faro Almirante Montt) lográndose también visualizar el borde costero oeste más cercano, frente a Puerto Natales. Entre el 27 y 28 de abril se realizó el censo de otoño, contándose esta vez con un bote inflable que permitió incluir el sector sur del canal Señoret hasta el faro Almirante Montt en la isla Cisnes, y el interior del fiordo Eberhard. Finalmente, el 7 de julio se realizó el censo de invierno; utilizando un bote inflable con el cual se recorrió el canal Señoret hasta el faro Almirante Montt por el sur y hasta la isla Guanaco por el norte, además del interior del fiordo Eberhard. En este último censo no se incluyó el fiordo Última Esperanza por problemas logísticos.

\section{c) Análisis de los datos}

Con los valores obtenidos de cada censo se realizaron estimaciones de riqueza específica, tanto estacional como para cada sector estudiado, utilizando el índice de diversidad de Margalef $(G)$ :

$$
G=\frac{S-1}{\ln N},
$$

siendo $\mathrm{S}=$ número de especies y $\mathrm{N}=$ número total de individuos (Magurran 1988).

El análisis de los datos para agrupamiento jerárquico y escalamiento multidimensional no métrico (MDS) de fechas de censos específicamente para aves se realizó con las rutinas CLUSTER y MDS del programa computacional PRIMER v6 (Clarke \& Gorley 2006), utilizando el índice de similitud de Bray-Curtis previa transformación de los datos de abundancia para cada censo a raíz cuarta. La contribución de especies a los porcentajes de similitud/disimilitud tanto dentro de cada grupo como entre grupos definidos con CLUSTER y MDS fue determinada con la rutina SIMPER de PRIMER.

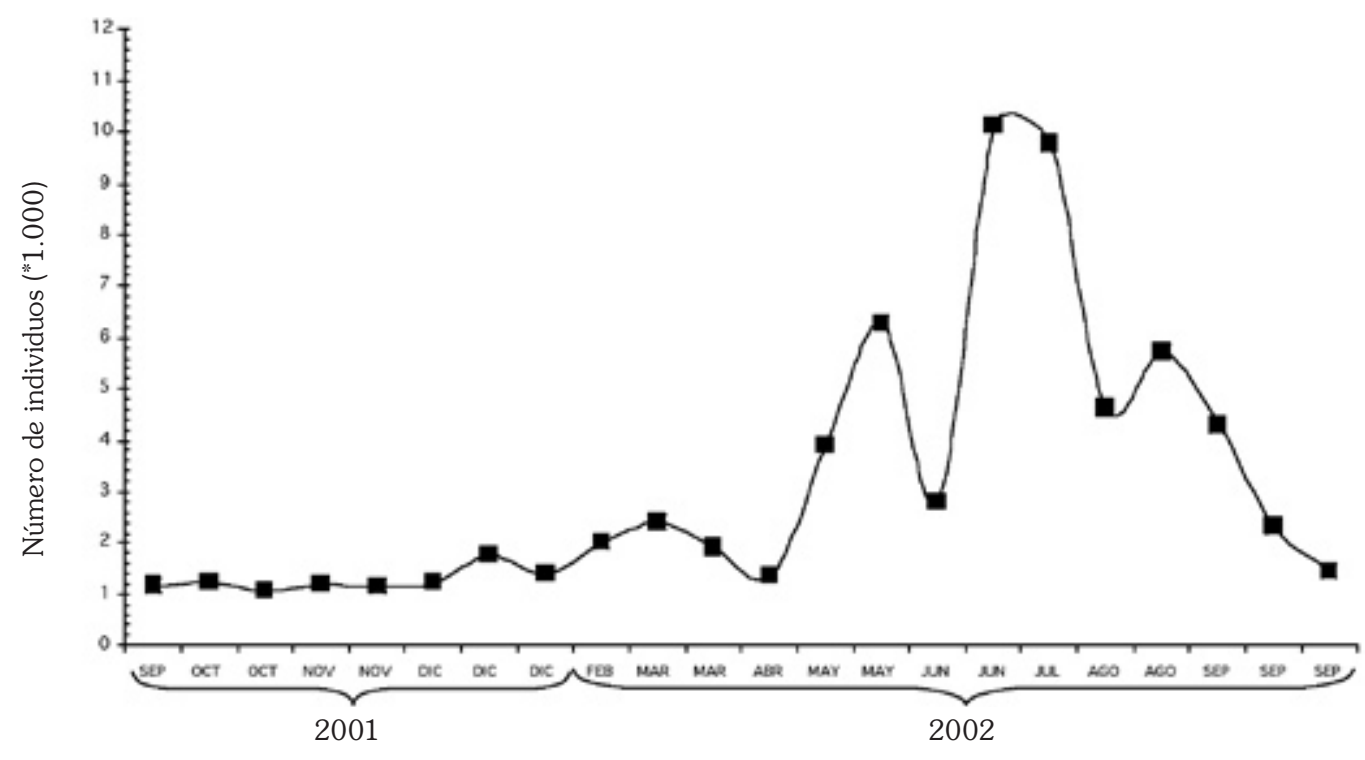

Fig. 2.- Número de ejemplares de aves censados durante el período de estudio en el fiordo Última Esperanza y canal Señoret. 


\section{RESULTADOS Y DISCUSIÓN}

En total, se registraron 33 especies de aves en el área de estudio; 3 de las cuales fueron terrestres (una no identificada) y las restantes especies acuáticas (Tabla 2). Este grupo se distribuyó en 9 órdenes y 11 familias, con un 34,4\% de herbívoros, 34,4\% de carnívoros, $18,7 \%$ de insectívoros-carnívoros y $12,5 \%$ de carroñeros.

La población total de aves registrada en el área de estudio durante los censos mensuales varió entre
1.084 individuos (censo de octubre 2001) y 10.139 (censo de junio 2002), disminuyendo nuevamente a 1.455 ejemplares en el censo de septiembre de 2002 (Fig. 2). Estos datos sugieren que el área de estudio sería utilizada por las aves acuáticas como área de invernada; coincidentemente cuando las lagunas y lagos interiores que usan en el período reproductivo se secan o congelan y los recursos alimentarios disminuyen. Esta situación coincide con datos de otros autores que sugieren áreas de invernada tanto en este canal (e.g. Markham 1971,

TABLA 2. Especies de aves registradas durante los censos regulares y estacionales realizados en diferentes sitios del Fiordo Última Esperanza y Canal Señoret entre septiembre de 2001 y septiembre de 2002. Se incluyen los hábitos tróficos de cada una de las especies registradas. $\mathrm{H}=$ herbívoro, $\mathrm{C}=$ carnívoro, $\mathrm{I}=$ insectívoro, $\mathrm{IC}=$ insectívoro- carnívoro, $\mathrm{Crr}=$ carroñero.

\begin{tabular}{|c|c|c|}
\hline & Nombre común & Hábito trófico \\
\hline \multicolumn{3}{|l|}{ Aves terrestres } \\
\hline Vultur gryphus & Cóndor & $\mathrm{Crr}$ \\
\hline Theristicus melanopis & Bandurria & IC \\
\hline \multicolumn{3}{|l|}{ Indeterminae } \\
\hline \multicolumn{3}{|l|}{ Aves acuáticas } \\
\hline Spheniscus magellanicus & Pingüino de Magallanes & C \\
\hline Phalacrocorax atriceps & Cormorán Imperial & $\mathrm{C}$ \\
\hline Phalacrocorax magellanicus & Cormorán de las Rocas & $\mathrm{C}$ \\
\hline Phalacrocorax olivaceus & Cormorán Negro & C \\
\hline Larus dominicanus & Gaviota Dominicana & C-Crr \\
\hline Larus scoresbii & Gaviota Austral & C-Crr \\
\hline Larus maculipennis & Gaviota Cahuil & $\mathrm{C}-\mathrm{Crr}$ \\
\hline Sterna hirundinacea & Gaviotín Sudamericano & C \\
\hline Catharacta chilensis & Salteador o skua & $\mathrm{C}$ \\
\hline Haematopus leucopodus & Pilpilén Austral & IC \\
\hline Haematopus ater & Pilpilén Negro & IC \\
\hline Phoenicopterus chilensis & Flamenco Chileno & IC \\
\hline Podiceps rolland & Pimpollo & IC \\
\hline Podiceps major & Huala Grande & IC \\
\hline Fulica armillata & Tagua común & $\mathrm{H}$ \\
\hline Chloephaga picta & Caiquén & $\mathrm{H}$ \\
\hline Chloephaga poliocephala & Canquén & $\mathrm{H}$ \\
\hline Chloephaga hybrida & Caranca & $\mathrm{H}$ \\
\hline Coscoroba coscoroba & Cisne Coscoroba & $\mathrm{H}$ \\
\hline Cygnus melancoryphus & Cisne Cuello Negro & $\mathrm{H}$ \\
\hline Lophonetta specularioides & Pato Juarjual & $\mathrm{C}$ \\
\hline Tachyeres pteneres & Pato Quetru No Volador & $\mathrm{C}$ \\
\hline Tachyeres patachonicus & Pato Quetru Volador & $\mathrm{C}$ \\
\hline Anas sibilatrix & Pato Real & $\mathrm{H}$ \\
\hline Anas flavirostris & Pato Jergón Chico & $\mathrm{H}$ \\
\hline Anas georgica & Pato Jergón Grande & $\mathrm{H}$ \\
\hline Anas platalea & Pato Cuchara & $\mathrm{C}$ \\
\hline Anas specularis & Pato Anteojillo & $\mathrm{C}$ \\
\hline Oxiura jamaicensis & Pato Rana de pico ancho & $\mathrm{H}$ \\
\hline Oxiura vittata & Pato Rana de pico delgado & $\mathrm{H}$ \\
\hline
\end{tabular}



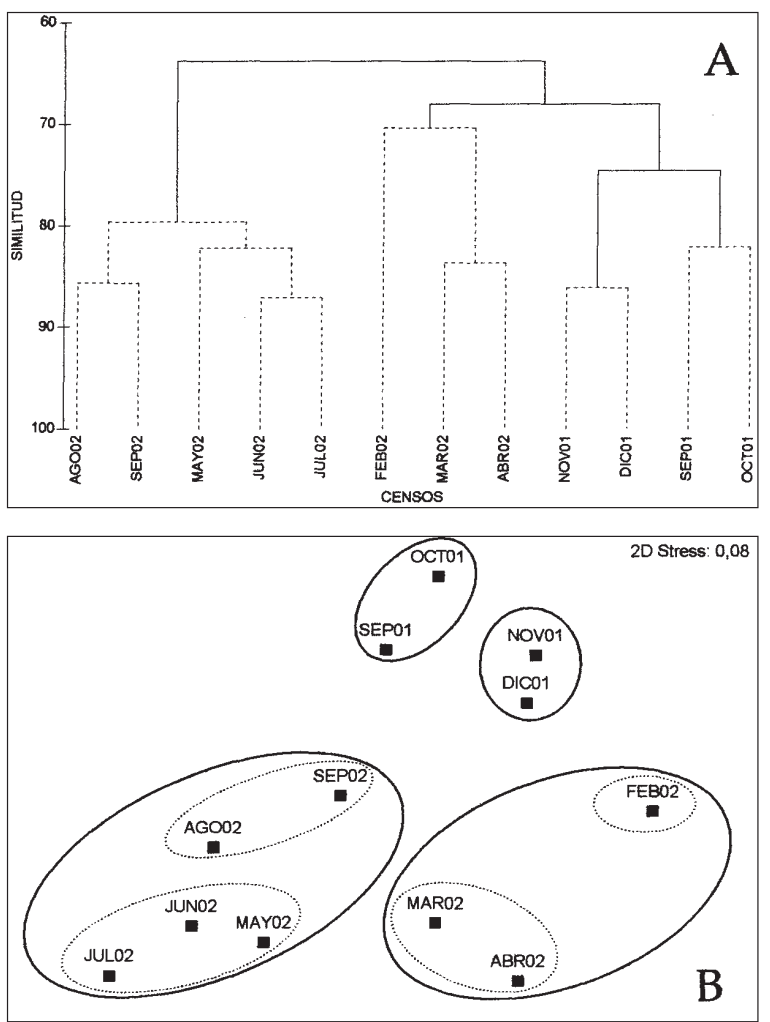

Fig. 3. Dendrograma (A) y escalamiento multidimensional MDS (B) para los datos de los censos promediados de aves obtenidos en el fiordo Última Esperanza y canal Señoret.

Vuilleumier 1997) como en otros canales y fiordos del archipiélago patagónico (e.g. Bernath 1965, Olrog 1948, Venegas 1994, Venegas \& Jory 1979). De acuerdo con los resultados obtenidos del análisis de agrupamiento y de escalamiento multidimensional (Fig. 3), existen evidencias para sostener que el ensamble de aves presentes en el área de estudio varía según la estación de año. El dendrograma de la figura 3 y el análisis de perfiles de similitud correspondiente (SIMPROF), indican la existencia de agrupaciones estadísticamente diferenciadas, específicamente el grupo conformado con los censos realizados principalmente en los meses de otoño-invierno versus los censos realizados en las estaciones de primavera-verano $(\pi=3,1 ; \mathrm{p}<$ $0,1)$. Una segunda diferenciación significativa se obtuvo dentro del grupo constituido por los censos de primavera-verano. Específicamente dentro de esta agrupación se diferenciaron significativamente del resto, los censos realizados entre febrero y abril (verano austral; $\pi=1,6 ; p<0,8$ ) y los de noviembre $y$ diciembre (fines de primavera e inicios de verano australes) en relación con los de septiembre y octubre (primavera; $\pi=1,9 ; \mathrm{p}<2,8$ ).

De acuerdo con el análisis de similitud, las especies que contribuyen con mayor porcentaje a la similitud dentro del grupo de censos realizados en las estaciones de primavera-verano (similitud promedio 82\%) son cormorán imperial, cisne de cuello negro, gaviota dominicana, caiquén y coscoroba. En la agrupación de los censos invernales las especies que contribuyen en mayor porcentaje a la similitud dentro del grupo (82\%) son la tagua, cormorán imperial, cisne de cuello negro, gaviota dominicana, coscoroba y flamenco chileno. Estos resultados demuestran la persistencia durante el año calendario en el área de estudio de especies tales como cormorán imperial, cisne de cuello negro, gaviota dominicana y coscoroba versus otras que parecen migrar hacia (e.g. tagua común, flamenco chileno) o desde (e.g. caiquén, cóndor, gaviota cahuil) el área de estudio durante el período invernal. Este comportamiento parece ser un patrón generalizado en los sistemas acuáticos de la región de Magallanes (C. Muñoz, com. pers.)

TABLA 3. Valores del número de especies de aves $(\mathrm{S})$ y abundancia total $(\mathrm{N})$ registrados durante 5 censos faunísticos realizados en 5 sectores geográficos del Fiordo Última Esperanza, región de Magallanes.

\begin{tabular}{|c|c|c|c|c|c|c|c|c|c|c|}
\hline \multirow[b]{3}{*}{ Sectores* } & \multicolumn{10}{|c|}{ Fecha de censo } \\
\hline & \multicolumn{2}{|c|}{19 septiembre } & \multicolumn{2}{|c|}{3 noviembre } & \multicolumn{2}{|c|}{$\begin{array}{c}28 \\
\text { enero }\end{array}$} & \multicolumn{2}{|c|}{$\begin{array}{c}27 \\
\text { abril }\end{array}$} & \multicolumn{2}{|c|}{$\begin{array}{c}9 \\
\text { mayo }\end{array}$} \\
\hline & $\mathrm{S}$ & $\mathrm{N}$ & S & $\mathrm{N}$ & $\mathrm{S}$ & $\mathrm{N}$ & $\mathrm{S}$ & $\mathrm{N}$ & S & $\mathrm{N}$ \\
\hline $\mathrm{A}$ & 10 & 383 & 6 & 408 & 9 & 531 & 11 & 1.371 & 14 & 1.939 \\
\hline B & 11 & 178 & 9 & 88 & 7 & 529 & 7 & 177 & 10 & 1.523 \\
\hline $\mathrm{C}$ & 9 & 517 & 7 & 687 & 11 & 1.332 & 2 & 40 & 7 & 383 \\
\hline D & 4 & 24 & 5 & 17 & 5 & 4 & 4 & 21 & 4 & 76 \\
\hline E & 6 & 14 & 3 & 13 & 2 & 4 & 0 & 0 & 0 & 0 \\
\hline
\end{tabular}

*Véase figura 1 para localización de los sectores 
Las especies más abundantes registradas en cada uno de los tres censos estacionales se indican en la tabla 3. En el censo general de verano (enero de 2002) se registró un total de 20 especies, las que congregaron 4.804 individuos. En esta época, la especie más abundante fue Larus dominicanus (gaviota dominicana) con 1.531 ejemplares (31,9\% del total). Las 14 especies restantes estuvieron representadas por abundancias inferiores a 100 individuos. Durante el censo de otoño (abril de 2002) se registraron 22 especies, que congregaron a 16.372 individuos. La especie más abundante fue Fulica armillata (tagua) con 6.635 ejemplares (40,5\% del total). Las restantes especies tuvieron una abundancia menor a 170 ejemplares (<1\%). En el censo general de invierno (julio de 2002) se registraron 7.000 individuos distribuidos en 21 especies. La especie más abundante fue nuevamente Fulica armillata (2.423 ejemplares; 34,5\%). Las restantes especies estuvieron representadas por menos de 100 individuos. Los resultados registrados en estos censos específicos concuerdan con las observaciones obtenidas de los censos mensuales.

El número de especies durante los censos mensuales varió entre 12 y 22 , con un promedio de 17 especies de aves para el período de estudio (Fig. 4). Los valores de riqueza encontrados durante los censos generales de verano, otoño e invierno fueron relativamente similares $(20,22$ y 21 especies, respectivamente). La riqueza específica $(G)$ calculada a partir de los datos de los censos generales fue de $\mathrm{G}=2,24$ para el verano, de $\mathrm{G}=2,16$ para otoño $\mathrm{y}$ de $\mathrm{G}=2,26$ para el invierno.

Para determinar una posible segregación de sitios utilizables por las especies que se registraron en los censos mensuales, el área general de trabajo se dividió arbitrariamente en 5 secciones (Fig.1) y para cada una de ellas se calculó la riqueza específica $(G)$ considerando 5 fechas de control (Tabla 4). Los valores obtenidos complementan además la variabilidad estacional registrada a partir de los censos mensuales.

La riqueza específica de los cinco sectores varió lo largo del año, con valores más elevados en el canal Señoret (A). En este sector, los valores fueron mayores en otoño, con 14 especies registradas y una población total de más de 1.900 aves (Tabla 4). Los valores para isla Guanaco (B) disminuyeron hacia el verano, con un incremento hacia el invierno, constituyéndose en la segunda área de mayor concentración de aves con más de 1.500 ejemplares distribuidos en 11 especies.

La bahía Delfines (C) es el sector que presentó una mayor variabilidad, con valores de $\mathrm{G}$ que variaron entre 1,39 y 0,27 durante el año. En este sector destacaron dos situaciones. Una fue la presencia

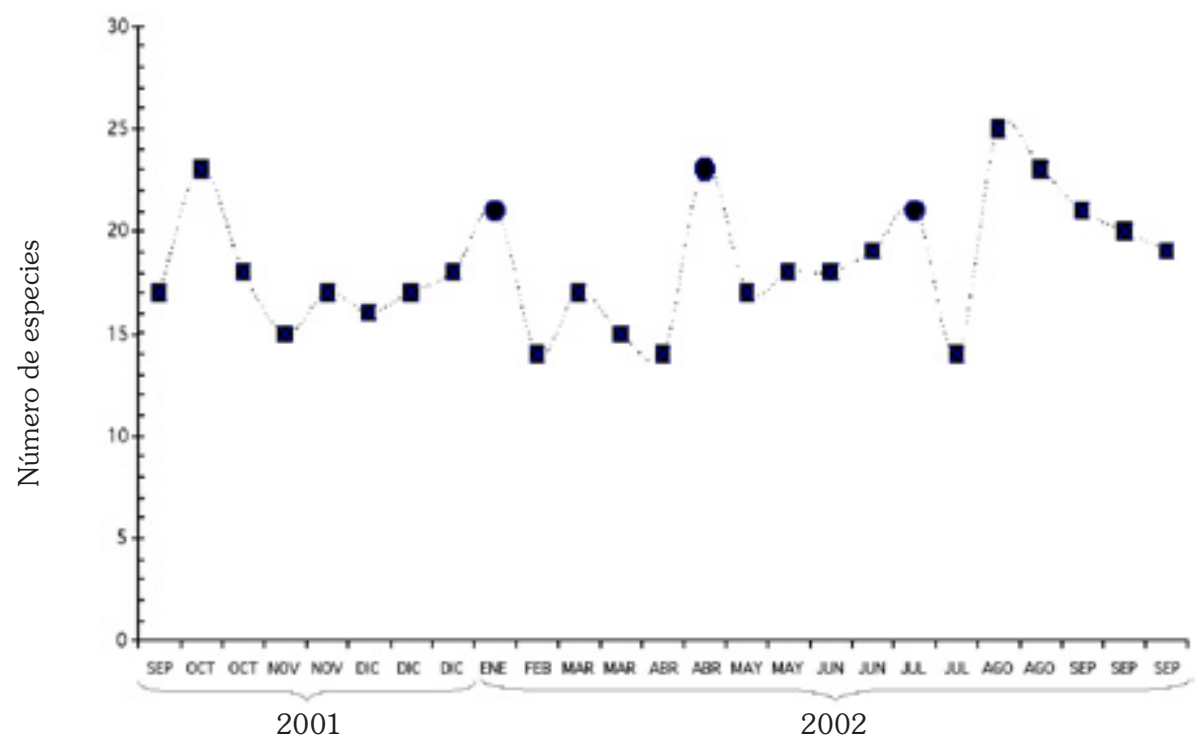

Fig. 4.- Número de especies de aves censadas en todo el período de muestreo en el fiordo Última Esperanza y canal Señoret. Se incluyen los valores de los censos generales $(\bullet)$ realizados en las estaciones de verano (ENE), otoño (ABR) e invierno (JUL). 
TABLA 4. Abundancia numérica de las principales especies de aves registradas en los censos generales correspondientes a verano (enero), otoño (abril) e invierno (julio) austral. Se incluye la representatividad relativa (\%) de cada especie en función del total censado.

\begin{tabular}{|c|c|c|c|c|c|c|}
\hline \multirow{4}{*}{$\begin{array}{l}N^{\circ} \text { total de individuos } \\
N^{\circ} \text { total de especies } \\
\text { Especies" }^{*}\end{array}$} & \multicolumn{2}{|c|}{ enero } & \multicolumn{2}{|c|}{ abril } & \multicolumn{2}{|c|}{ julio } \\
\hline & \multicolumn{2}{|c|}{4.804} & \multicolumn{2}{|c|}{16.372} & \multicolumn{2}{|c|}{7.000} \\
\hline & \multicolumn{2}{|c|}{20} & \multicolumn{2}{|c|}{22} & \multicolumn{2}{|c|}{21} \\
\hline & $\mathrm{N}$ & $\%$ & & $\%$ & & $\%$ \\
\hline Tagua & 0 & - & 6.635 & 40,5 & 2.423 & 34,5 \\
\hline Cisne de cuello negro & 667 & 13,9 & 3.291 & 20,1 & 1.161 & 16,6 \\
\hline Gaviota dominicana & 1.531 & 31,9 & 1.741 & 10,6 & 766 & 10,9 \\
\hline Cormorán Imperial & 1.246 & 25.8 & 1.472 & 9,0 & 613 & 8,8 \\
\hline Cisne coscoroba & 0 & - & 646 & 3,9 & 352 & 5,0 \\
\hline Cormorán de las rocas & 112 & 2,3 & 178 & 1,1 & 168 & 2,4 \\
\hline Caiquén & 415 & 8,6 & 260 & 1,6 & 0 & - \\
\hline Gaviota cahuil & 306 & 6,4 & 0 & - & 0 & - \\
\hline Flamenco chileno & 0 & - & 0 & - & 432 & 6,2 \\
\hline Pato rana & 0 & - & 0 & - & 400 & 5,7 \\
\hline Pato quetru volador & 0 & - & 0 & - & 231 & 3,3 \\
\hline
\end{tabular}

*Para nombres científicos refiérase a tabla 2

de las colonias de cormoranes que permanecieron ocupadas en primavera y verano, con un desplazamiento al área del canal Señoret en otoño. Por otra parte, en la ensenada que se forma en la costa norte de bahía Delfines se concentraron grandes cantidades de cisnes de cuello negro y flamencos chilenos en otoño e invierno, estando ausentes en las estaciones de primavera y verano. En el caso del cisne de cuello negro, el aumento otoñal tendría relación con las migraciones locales y quizá también regionales, de ejemplares de esta especie hacia los canales marinos, luego de terminado el período reproductivo; tanto para el período de cambio de plumaje (muda), como para alimentarse, dado el desecamiento de lagos y lagunas interiores a fines del verano y/o congelamiento de ellas ya a mediados de otoño (Garay et al. 1991). Es posible también que ocurran desplazamientos de cormoranes en la oscuridad durante otoño e invierno (i.e. antes de las 10:00 AM y después de las 16:00 PM). En efecto, al interior del fiordo frente a barranca Lobos, se observó una pared rocosa con abundante presencia de fecas, lo cual sugiere un sitio de uso activo aunque raramente se registraron cormoranes durante el día.

Hacia el norte de punta Barrosa (D), se observó una disminución de la riqueza específica ( 2 a 4 especies) durante los censos de primavera y verano. Por su parte, el sector más extremo del fiordo, cercano al monte Balmaceda (E), se caracterizó por el menor número de especies en comparación con los restantes sectores.

Los resultados descritos previamente podrían estar relacionados tanto con la topografía de los distintos sectores como con los tipos de alimentación y reproducción de las diversas especies registradas. La primera mitad del fiordo, con zonas onduladas y bajas y una vegetación estepárica de matorral, sería apropiada para la reproducción de la mayor parte de las aves (Anseriformes y Podiceps) presentes en el área, mientras que las ensenadas protegidas y someras de la zona costera con presencia de vegetación acuática y organismos marinos del intermareal e infralitoral parecen ser adecuadas para su alimentación. Se debe considerar también el hecho que al fiordo Última Esperanza desemboca el fiordo Eberhard, el cual es utilizado intensivamente para actividades de salmonicultura. Es posible que la existencia de un suministro adicional de alimentos en estas aguas permita una mayor carga de aves, favoreciendo una mayor riqueza de especies.

Por otra parte, en la segunda mitad del fiordo, donde la topografía tanto sobre como bajo el agua es más abrupta, la riqueza específica resultó ser menor. Este tramo resultó apto como área de uso permanente sólo para un par de especies piscívoras, las que no requerirían obligadamente costas someras para la búsqueda de su alimento. Además de la ausencia de ensenadas someras, se debe considerar la diferencia en la calidad de las aguas. Las aguas 
más cercanas al monte Balmaceda (sectores E y D) tienen menor carga salina por el desagüe del río serrano y de otros que bajan desde los glaciares del monte Balmaceda, aspecto que podría generar una menor cantidad de nutrientes en comparación con las más salinas cercanas al canal Señoret. Asimismo, en invierno las aguas del sector más cercano al Balmaceda tienden a congelarse.

\section{CONCLUSIONES}

1. De acuerdo a la información obtenida, se puede concluir que el área del canal Señoret y fiordo Última Esperanza, es una zona importante de uso para al menos 33 especies de aves.

2. De las 18 especies de aves seleccionadas para un seguimiento anual, 10 resultaron ser residentes y 8 visitantes estacionales; otras 4 especies no seleccionadas, fueron registradas regularmente, indicando residencia.

3. Entre las aves, las 10 especies residentes son: cóndor, coscoroba, cisne cuello negro, quetru no volador, quetru volador, cormorán imperial, cormorán de las rocas, cormorán negro, caiquén y huala. Las restantes 4 incluyen a la gaviota dominicana, gaviota austral, salteador y pato juarjual. Se debe destacar que una parte de la población global de estas aves ingresa al sector durante el invierno, elevando los valores basales de abundancia de la población residente.

4. Las 8 especies de aves visitantes regulares son: la bandurria y el canquén que llegan para su período reproductivo en primavera, y el pingüino, cormorán negro, flamenco chileno, tagua, pato rana de pico ancho y pato rana de pico delgado, que llegan para el período de muda y/o invernada. Para establecer el estatus de residencia de las otras especies de aves, es necesario un estudio más detallado y con mayor frecuencia de controles.

5. En el caso de la muda e invernada, dado el elevado número de cisnes y taguas, es posible que la inmigración no sea solamente de la población local de las lagunas interiores de la provincia de Última Esperanza, sino que también podría ser regional, incluyendo parte de la Patagonia argentina, situación que ya fue planteada por Markham (1971) y Vuilleumier (1997).

6. Respecto a las áreas de uso, hay 4 colonias reproductivas de cormoranes y una de bandurria. Por otra parte, la vegetación costera del área permitiría la nidificación de las aves menores mientras que los acantilados serían ocupados para los mismos fines por los cóndores.

7. Los cormoranes y otras aves utilizan roqueríos y áreas costeras en otoño e invierno en el canal Señoret y el sector más próximo del fiordo Última Esperanza.

8. El extremo sur del fiordo Última Esperanza y canal Señoret en particular, presentan los mayores valores de riqueza específica, siendo una zona de concentración de aves durante la época de muda en otoño y como área de alimentación y supervivencia durante el período de otoño e invierno. Algunas especies migran desde otras zonas, como es el caso de los flamencos.

9. Las especies residentes más abundantes fueron cormorán imperial, cisne de cuello negro, cormorán de las rocas, coscoroba y gaviota dominicana. Entre las especies visitantes regulares, las más abundantes fueron tagua, flamenco, pato rana y gaviota cáhuil.

10. Es importante destacar que en el área de estudio habitan 5 especies con problemas de conservación, de acuerdo al siguiente criterio:

a) En Peligro: coscoroba;

b) Vulnerables: cóndor, cisne de cuello negro, flamenco chileno;

c) Inadecuadamente Conocido: quetru volador.

Todas estas especies utilizan el área de estudio para su supervivencia invernal y, exceptuando al flamenco, los demás tienen lugares específicos de reproducción en primavera, período vital para su sobrevivencia como especies. El flamenco es una especie que no nidifica en la región, haciéndolo en el extremo norte de Chile y en zonas elevadas de la Patagonia argentina (Fjelds \& Krabbe 1990). Es probable que cuando las lagunas altiplánicas se congelen, parte de la población se desplace a invernar en las áreas sur-austral. Su período de estadía en Magallanes coincide con lo descrito para Chiloé (Von Meyer \& Espinosa 1998).

11. Los resultados obtenidos sugieren que el sistema marino estudiado contiene elementos de gran importancia para la mantención de los procesos vitales de una importante cantidad de especies de aves silvestres, las cuales por sus procesos migracionales de tipo estacional parecen ser elementos 
de conexión dentro de un ecosistema mucho mayor y complejo.

12. Las particularidades faunísticas observadas y registradas para todo el sector estudiado, en conjunto con las presiones de tipo antrópica a que se está sometiendo el sistema en su conjunto, recomiendan la necesidad de iniciar el estudio de medidas de protección adecuadas para el sistema.

\section{AGRADECIMIENTOS}

Este estudio contó durante todo su desarrollo con el apoyo logístico de la familia Álvarez-Rodríguez de Puerto Natales, representada por el Gerente Comercial de Turismo 21 de Mayo Sr. Víctor Álvarez. También se recibió el apoyo proporcionado por los siguientes socios de la Cámara de Turismo de Puerto Natales: Hotel Los Glaciares, Hostal Indigo, Hotel Luckowieck, Hospedaje La Cumbre, Restaurante La Tranquera y Café Rincón del Tata. Un especial agradecimiento a Víctor Álvarez, por su iniciativa y apoyo desinteresado para realizar este trabajo. Expresamos nuestros agradecimientos al Capitán y a la tripulación del yate/motor "Alberto de Agostini" por la colaboración prestada durante todas las actividades de terreno. Agradecemos el apoyo voluntario brindado en algunos controles por la Srta. Javiera Cárdenas (Universidad de Magallanes) y por el Sr. Ricardo Guineo (Liceo Experimental de la Universidad de Magallanes, Punta Arenas). También agradecemos a nuestros colegas Cristian Muñoz y Daniel-W Antunez (Laboratorio de Hidrobiología, Instituto de la Patagonia) por la colaboración en la recopilación de información y confección de la figura 1.

\section{LITERATURA CITADA}

Bernath, E. L. 1965. Observations in southern Chile in the southern hemisphere autumn. Auk 82: 95-101

Clarke, K. R. \& R. N. Gorley 2006. PRIMER v6: User Manual/Tutorial. PRIMER-E Ltd. 190 pp.

Fjelds, J. \& N. Krabbe 1990. Birds of the high Andes. Zoological Museum, University of Copenhagen and Apollo Books, Svendborg.

Garay, G., W. Johnson \& W. Franklin 1991. Relative abundance of aquatic birds and their use of wetlands in the Patagonia of Southern Chile. Revista Chilena de Historia Natural. 64: 127-137.

Gibbons, J., J. Capella, R. Matus \& L. Guzmán 1998. Ballenas jorobadas, Megaptera novaeangliae (Balanopteridae), en los canales patagónicos de Chile. Anales Instituto Patagonia. Serie Cs. Nat. (Chile) 26: 69-75.

Jara, F. 2005. Environmental considerations and conflicts derived from the development of investment projects on the coastal border in Southern Chile. En: Hellman \& Araya (eds.): Chile litoral: Diálogo científico sobre los ecosistemas costeros. pp. 101-110. FLACSO-Chile.

Magurran, A. E. 1988. Ecological diversity and its measurement. University Press, Cambridge. $179 \mathrm{pp}$.

Markham, B. J. 1971. Censo invernal de cisnes y flamencos en Magallanes. Anales Instituto Patagonia (Chile) 2:146-157.

Olrog, C. C. 1948. Observaciones sobre la avifauna de Tierra del Fuego y Chile. Acta Zool. Lilloana 5:437-531.

Pisano, E. 1977. Fitogeografía de Fuego-Patagonia chilena I.- Comunidades vegetales entre las latitudes $52^{\circ}$ y $56^{\circ} \mathrm{S}$. Anales Instituto Patagonia (Chile) 8: 121-250.

Sielfeld, W. \& C. Venegas, 1980. Observaciones de delfínidos en los canales australes de Chile. Anales Instituto Patagonia (Chile), 9: $145-151$.

Soto, D. \& F. Norambuena 2005. Monitores ambientales para evaluar efectos de actividades productivas en el borde costero y mares interiores de las regiones X y XI: el caso de la salmonicultura. En: Hellman \& Araya (eds.): Chile litoral: Diálogo científico sobre los ecosistemas costeros. pp. 101-110. FLACSO-Chile.

Venegas, C. 1994. Aves de Magallanes. Ed. Universidad de Magallanes, $158 \mathrm{pp}$.

Venegas, C. \& J. Jory 1979. Guía de campo para las aves de Magallanes. Serie Monografías $\mathrm{N}^{\circ} 11$ Pub. Inst. de la Patagonia, Punta Arenas, Chile. 253 pp.

Venegas, C. J. Gibbons, A. Aguayo, W. Sielfeld, J. Acevedo, N. Amado, J. Capella, G. Guzmán \& C. Valenzuela 2002. Distribución y 
abundancia de lobos marinos (Pinnipedia : Vuilleumier, F. 1997. A large autumn concentraOtariidae) en la región de Magallanes, Chile. Anales Instituto Patagonia. Serie Cs. Nat. (Chile) 30: 67-82.

Von Meyer, A. \& L. Espinosa. 1998. Situación del Flamenco chileno (Phoenicopterus chilensis) en Chiloé y sur de la provincia de Llanquihue. Bol. Chileno de Ornitol. 5: 16-20. tion of swans (Cignus melancoryphus and Coscoroba coscoroba) and other waterbirds at Puerto Natales, Magallanes, Chilean Patagonia, and its significance for swan and waterfowl conservation. Ornitol. Neotrop. 8: $1-5$. 